\title{
Proceedings of the Annual \\ Plenary Session on Regenerative Medicine (PAPRM)
}

JSRM Code: 010020700008

\section{Human Monoclonal antibodies - A dual advantaged weapon to tackle cancer and viruses}

\author{
Kurosawa $\mathrm{G}^{*}$
}

Abstract

\begin{abstract}
Human monoclonal antibodies (mAbs) are powerful tools as pharmaceutical agents to tackle cancer and infectious diseases. Antibodies (Abs) are present in blood at the concentration of $10 \mathrm{mg} / \mathrm{ml}$ and play a vital role in humoral immunity. Many therapeutic Abs have been reported since early 1980 s. Human mAb technology was not available at that time and only the hybridoma technology for making mouse mAbs had been well established. In order to avoid various potential problems associated with use of mouse proteins, two different technologies to make human/mouse chimeric $A b$ as well as humanized $A b$ were developed crossing the various hurdles for almost twenty years and mAb based drugs such as rituximab, anti-CD20 Ab, and trastuzumab, anti-HER2 $\mathrm{Ab}$, have been approved by the US Food and Drug Administration (FDA) for treatment of non-Hodgkin's lymphoma and breast cancer in 1997 and 1998, respectively. These drugs are well recognized and accepted by clinicians for treatment of patients. The clinical outcome of the treatment with $m A b$ has strongly encouraged the researchers to develop much more refined mAbs. In addition to chimeric $A b$ and humanized $A b$, now human mAbs can be produced by two technologies. The first is transgenic mice that produce human Abs and the second is human Ab libraries using phage-display system.
\end{abstract}

Until now, several hundreds of mAbs against several tens of antigens (Ags) have been developed and subjected to clinical examinations. While many Abs have been approved as therapeutic agents against hematological malignancies, the successful mAbs against solid tumors are still limited. However, many researchers have suggested that developing potential mAbs agents should be possible and incurable cancers may become curable within another decade. Though it is hard to say explicitly that this prediction is correct, a passion for this development should be worth supporting to lead to a successful outcome which will lead to patient benefits.

Our institute has been working on newer technologies for developing human mAbs. For the comprehensive isolation of mAbs, we have developed a method known as ICOS (isolation of antigen-antibody complexes through organic solvent) method ${ }^{[1]}$, in which phage particles of an antibody (Ab) library are mixed with living cells which leads to formation of antigen ( $\mathrm{Ag})-\mathrm{Ab}$ complexes on the cell surface. The mixture is then overlaid on organic solution and phage bound to cells are recovered from the precipitate after centrifugation ${ }^{[1]}$. Further, for the rapid identification of Antigens (Ags) recognized by the several mAbs, we have developed the GFC [grouping of clones by flow cytometry (FCM)] method and the SITE (simultaneous identification of clones through three dimensional ELISA) method (2). These methods helped us to identify 24 distinct tumour associated antigens (TAAs) that are associated with several carcinomas and we were able to isolate 432 human mAbs that specifically bound to one of the $24 \mathrm{TAAs}^{[2]}$, making these mAbs potential therapeutic agents against a variety of cancers.

To understand the way forward from our perspectives, a clear knowledge on the following are indispensable

- Differences between human Abs and the other Abs such as mouse, chimeric and humanized Abs.

- Differences between Abs and small molecules.

- Differences between Abs and vaccination.

- How to materialize best agents using human mAbs.

References

1. Akahori $Y$, Kurosawa $G$, Sumitomo $M$, Morita $M$, Muramatsu C, Eguchi K, Tanaka M, Suzuki K, Sugiura M, Iba Y, Sugioka A, Kurosawa Y. Isolation of antigen/antibody complexes through organic solvent (ICOS) method. Biochem Biophys Res Commun. 2009;378(4):832-5.

2. Kurosawa G, Sumitomo M, Akahori $Y$, Matsuda K, Muramatsu C, Takasaki A, Iba Y, Eguchi K, Tanaka M, Suzuki K, Morita M, Sato N, Sugiura M, Sugioka A, Hayashi N, Kurosawa Y. Methods for comprehensive identification of membrane proteins recognized by a large number of monoclonal antibodies. J Immunol Methods. 2009;351(1-2):1-12. 


\title{
Proceedings of the Annual \\ Plenary Session on Regenerative Medicine (PAPRM)
}

JSRM Code: 010020700009

ISSN $0973-7154$

\section{The intricacies of pluripotency}

\author{
Bhartiya $D^{*}$
}

\begin{abstract}
Pluripotent stem cells derived from the inner cell mass of a blastocyst or obtained by reprogramming somatic cells using 'Yamanaka factors' are termed human embryonic stem (hES) and induced pluripotent stem (iPS) cells respectively. These stem cells have been reported to have maximum potential for regenerative medicine as they can differentiate into all three germ layers and 200 odd cell types in the body. However, lately it is being realized that hES cells differentiate into their fetal counterparts and whether they can regenerate adult organs requires more research. Simultaneously several autologous adult stem cell trials undertaken using blood cells isolated by various methods (adult stem cells are never enriched) conducted worldwide have resulted in mixed results. Whatever, beneficial effect observed in small pilot studies disappears once double blinded trials are undertaken. It is now believed that the beneficial effect observed is a growth factor effect rather than true regeneration.

Against this background, a novel pluripotent stem cell has been reported which exist in adult body organs termed very small embryonic-like stem cells (VSELs). These cells were described for the first time by Professor Ratajczak's group

at the University of Louisville, USA. It is believed that when primordial germ cells (or their precursors) migrate along the dorsal mesentery to settle in the gonadal ridge, indeed settle in all the developing organs and survive throughout life. They are the stem cells sitting on the top of hierarchy of adult stem cells in the body, maintaining life-long homeostasis, serving as a back-up pool for adult stem cells and are possibly the embryonic remnants that get transformed into cancer stem cells under certain yet not well understood changes in the somatic microenvironment. They are relatively quiescent in nature and give rise to the progenitors which divide rapidly, form cysts (i.e. undergo clonal expansion), undergo further differentiation into tissue specific cells and are easily mobilized in case of any injury.

Similar to hES and iPS cells, VSELs are pluripotent and may prove to be better than them in a clinic because (i) they can be isolated from autologous source and thus immune rejection issues do not exist (ii) they do not form teratoma and (iii) as they are pluripotent stem cells in adult organs, they will regenerate them efficiently. Various examples have been reported to demonstrate the potential of VSELs.
\end{abstract}

